\title{
New general integral inequalities for quasi-geometrically convex functions via fractional integrals
}

Imdat İşcan*

"Correspondence:

imdat.iscan@giresun.edu.tr Department of Mathematics,

Faculty of Sciences and Arts, Giresun University, Giresun, 28100, Turkey

\begin{abstract}
In this paper, the author introduces the concept of the quasi-geometrically convex functions, gives Hermite-Hadamard's inequalities for GA-convex functions in fractional integral forms and defines a new identity for fractional integrals. By using this identity, the author obtains new estimates on generalization of Hadamard et al. type inequalities for quasi-geometrically convex functions via Hadamard fractional integrals.

MSC: Primary 26A33; 26A51; secondary 26D15
\end{abstract}

Keywords: quasi-geometrically convex function; Hermite-Hadamard-type inequalities; Hadamard fractional integrals

\section{Introduction}

Let a real function $f$ be defined on some nonempty interval $I$ of a real line $\mathbb{R}$. The function $f$ is said to be convex on $I$ if inequality

$$
f(t x+(1-t) y) \leq t f(x)+(1-t) f(y)
$$

holds for all $x, y \in I$ and $t \in[0,1]$.

We recall that the notion of quasi-convex function generalizes the notion of convex function. More exactly, a function $f:[a, b] \subset \mathbb{R} \rightarrow \mathbb{R}$ is said to be quasi-convex on $[a, b]$ if

$$
f(t x+(1-t) y) \leq \max \{f(x), f(y)\}
$$

for all $x, y \in[a, b]$ and $t \in[0,1]$. Clearly, any convex function is a quasi-convex function. Furthermore, there exist quasi-convex functions which are not convex (see [1]).

The following inequalities are well known in the literature as the Hermite-Hadamard inequality, the Ostrowski inequality and the Simpson inequality, respectively.

Theorem 1.1 Let $f: I \subseteq \mathbb{R} \rightarrow \mathbb{R}$ be a convex function defined on the interval I of real numbers, and let $a, b \in I$ with $a<b$. The following double inequality holds:

$$
f\left(\frac{a+b}{2}\right) \leq \frac{1}{b-a} \int_{a}^{b} f(x) d x \leq \frac{f(a)+f(b)}{2} .
$$

O2013 Işcan; licensee Springer. This is an Open Access article distributed under the terms of the Creative Commons Attribution License (http://creativecommons.org/licenses/by/2.0), which permits unrestricted use, distribution, and reproduction in any medium, provided the original work is properly cited. 
Theorem 1.2 Let $f: I \subseteq \mathbb{R} \rightarrow \mathbb{R}$ be a mapping differentiable in $I^{\circ}$, the interior of $I$, and let $a, b \in I^{\circ}$ with $a<b$. If $\left|f^{\prime}(x)\right| \leq M, x \in[a, b]$, then the following inequality holds:

$$
\left|f(x)-\frac{1}{b-a} \int_{a}^{b} f(t) d t\right| \leq \frac{M}{b-a}\left[\frac{(x-a)^{2}+(b-x)^{2}}{2}\right]
$$

for all $x \in[a, b]$.

Theorem 1.3 Let $f:[a, b] \rightarrow \mathbb{R}$ be a four times continuously differentiable mapping on $(a, b)$ and $\left\|f^{(4)}\right\|_{\infty}=\sup _{x \in(a, b)}\left|f^{(4)}(x)\right|<\infty$. Then the following inequality holds:

$$
\left|\frac{1}{3}\left[\frac{f(a)+f(b)}{2}+2 f\left(\frac{a+b}{2}\right)\right]-\frac{1}{b-a} \int_{a}^{b} f(x) d x\right| \leq \frac{1}{2,880}\left\|f^{(4)}\right\|_{\infty}(b-a)^{4} .
$$

The following definitions are well known in the literature.

Definition 1.1 $([2,3])$ A function $f: I \subseteq(0, \infty) \rightarrow \mathbb{R}$ is said to be GA-convex (geometricarithmetically convex) if

$$
f\left(x^{t} y^{1-t}\right) \leq t f(x)+(1-t) f(y)
$$

for all $x, y \in I$ and $t \in[0,1]$.

Definition $1.2([2,3])$ A function $f: I \subseteq(0, \infty) \rightarrow(0, \infty)$ is said to be GG-convex (called in [4] a geometrically convex function) if

$$
f\left(x^{t} y^{1-t}\right) \leq f(x)^{t} f(y)^{(1-t)}
$$

for all $x, y \in I$ and $t \in[0,1]$.

We will now give definitions of the right-hand side and left-hand side Hadamard fractional integrals which are used throughout this paper.

Definition 1.3 Let $f \in L[a, b]$. The right-hand side and left-hand side Hadamard fractional integrals $J_{a^{+}}^{\alpha} f$ and $J_{b^{-}}^{\alpha} f$ of order $\alpha>0$ with $b>a \geq 0$ are defined by

$$
J_{a+}^{\alpha} f(x)=\frac{1}{\Gamma(\alpha)} \int_{a}^{x}\left(\ln \frac{x}{t}\right)^{\alpha-1} f(t) \frac{d t}{t}, \quad a<x<b
$$

and

$$
J_{b-}^{\alpha} f(x)=\frac{1}{\Gamma(\alpha)} \int_{x}^{b}\left(\ln \frac{t}{x}\right)^{\alpha-1} f(t) \frac{d t}{t}, \quad a<x<b,
$$

respectively, where $\Gamma(\alpha)$ is the Gamma function defined by $\Gamma(\alpha)=\int_{0}^{\infty} e^{-t} t^{\alpha-1} d t$ (see [5]).

In recent years, many authors have studied error estimations for Hermite-Hadamard, Ostrowski and Simpson inequalities; for refinements, counterparts, generalization see [4, 6-20]. 
In this paper, the concept of the quasi-geometrically convex function is introduced, Hermite-Hadamard's inequalities for GA-convex functions in fractional integral forms are established, and a new identity for Hadamard fractional integrals is defined. By using this identity, author obtains a generalization of Hadamard, Ostrowski and Simpson type inequalities for quasi-geometrically convex functions via Hadamard fractional integrals.

\section{Main results}

Let $f: I \subseteq(0, \infty) \rightarrow \mathbb{R}$ be a differentiable function on $I^{\circ}$, the interior of $I$, throughout this section we will take

$$
\begin{aligned}
I_{f}(x, \lambda, \alpha, a, b) & \\
= & (1-\lambda)\left[\ln ^{\alpha} \frac{x}{a}+\ln ^{\alpha} \frac{b}{x}\right] f(x)+\lambda\left[f(a) \ln ^{\alpha} \frac{x}{a}+f(b) \ln ^{\alpha} \frac{b}{x}\right] \\
& -\Gamma(\alpha+1)\left[J_{x-}^{\alpha} f(a)+J_{x+}^{\alpha} f(b)\right],
\end{aligned}
$$

where $a, b \in I$ with $a<b, x \in[a, b], \lambda \in[0,1], \alpha>0$ and $\Gamma$ is the Euler Gamma function.

Definition 2.1 A function $f: I \subseteq(0, \infty) \rightarrow \mathbb{R}$ is said to be quasi-geometrically convex on $I$ if

$$
f\left(x^{t} y^{1-t}\right) \leq \sup \{f(x), f(y)\},
$$

for any $x, y \in I$ and $t \in[0,1]$.

Remark 2.1 Clearly, any GA-convex and geometrically convex functions are quasigeometrically convex functions. Furthermore, there exist quasi-geometrically convex functions which are neither GA-convex nor geometrically convex. In that context, we point out an elementary example. The function $f:(0,4] \rightarrow \mathbb{R}$,

$$
f(x)= \begin{cases}1, & x \in(0,1] \\ (x-2)^{2}, & x \in[1,4]\end{cases}
$$

is neither GA-convex nor geometrically convex on $(0,4]$, but it is a quasi-geometrically convex function on $(0,4]$.

Proposition 2.1 If $f: I \subseteq(0, \infty) \rightarrow \mathbb{R}$ is convex and nondecreasing, then it is quasigeometrically convex on $I$.

Proof This follows from

$$
\begin{aligned}
f\left(x^{t} y^{1-t}\right) & \leq f(t x+(1-t) y) \\
& \leq t f(x)+(1-t) f(y) \leq \sup \{f(x), f(y)\}
\end{aligned}
$$

for all $x, y \in I$ and $t \in[0,1]$. 
Proposition 2.2 Iff: $I \subseteq(0, \infty) \rightarrow \mathbb{R}$ is quasi-convex and nondecreasing, then it is quasigeometrically convex on I. If $: I \subseteq(0, \infty) \rightarrow \mathbb{R}$ is quasi-geometrically convex and nonincreasing, then it is quasi-convex on $I$.

Proof These conclusions follows from

$$
f\left(x^{t} y^{1-t}\right) \leq f(t x+(1-t) y) \leq \sup \{f(x), f(y)\}
$$

and

$$
f(t x+(1-t) y) \leq f\left(x^{t} y^{1-t}\right) \leq \sup \{f(x), f(y)\}
$$

for all $x, y \in I$ and $t \in[0,1]$, respectively.

Hermite-Hadamard's inequalities can be represented for GA-convex functions in fractional integral forms as follows.

Theorem 2.1 Let $f: I \subseteq(0, \infty) \rightarrow \mathbb{R}$ be a function such that $f \in L[a, b]$, where $a, b \in I$ with $a<b$. Iff is a GA-convex function on $[a, b]$, then the following inequalities for fractional integrals hold:

$$
f(\sqrt{a b}) \leq \frac{\Gamma(\alpha+1)}{2\left(\ln \frac{b}{a}\right)^{\alpha}}\left\{J_{a+}^{\alpha} f(b)+J_{b-}^{\alpha} f(a)\right\} \leq \frac{f(a)+f(b)}{2}
$$

with $\alpha>0$.

Proof Since $f$ is a GA-convex function on $[a, b]$, we have for all $x, y \in[a, b]$ (with $t=1 / 2$ in inequality (1)),

$$
f(\sqrt{x y}) \leq \frac{f(x)+f(y)}{2} .
$$

Choosing $x=a^{t} b^{1-t}, y=b^{t} a^{1-t}$, we get

$$
f(\sqrt{a b}) \leq \frac{f\left(a^{t} b^{1-t}\right)+f\left(b^{t} a^{1-t}\right)}{2} .
$$

Multiplying both sides of (4) by $t^{\alpha-1}$, then integrating the resulting inequality with respect to $t$ over $[0,1]$, we obtain

$$
\begin{aligned}
f(\sqrt{a b}) & \leq \frac{\alpha}{2}\left\{\int_{0}^{1} f\left(a^{t} b^{1-t}\right) d t+\int_{0}^{1} f\left(b^{t} a^{1-t}\right) d t\right\} \\
& =\frac{\alpha}{2}\left\{\int_{a}^{b}\left(\frac{\ln b-\ln u}{\ln b-\ln a}\right)^{\alpha-1} f(u) \frac{d u}{u \ln \frac{b}{a}}+\int_{a}^{b}\left(\frac{\ln u-\ln a}{\ln b-\ln a}\right)^{\alpha-1} f(u) \frac{d u}{u \ln \frac{b}{a}}\right\} \\
& =\frac{\alpha \Gamma(\alpha)}{2\left(\ln \frac{b}{a}\right)^{\alpha}}\left\{J_{a+}^{\alpha} f(b)+J_{b-}^{\alpha} f(a)\right\} \\
& =\frac{\Gamma(\alpha+1)}{2\left(\ln \frac{b}{a}\right)^{\alpha}}\left\{J_{a+}^{\alpha} f(b)+J_{b-}^{\alpha} f(a)\right\},
\end{aligned}
$$

and the first inequality is proved. 
For the proof of the second inequality in (3), we first note that if $f$ is a convex function, then for $t \in[0,1]$, it yields

$$
f\left(a^{t} b^{1-t}\right) \leq t f(a)+(1-t) f(b)
$$

and

$$
f\left(b^{t} a^{1-t}\right) \leq t f(b)+(1-t) f(a) .
$$

By adding these inequalities, we have

$$
f\left(a^{t} b^{1-t}\right)+f\left(b^{t} a^{1-t}\right) \leq f(a)+f(b) .
$$

Then multiplying both sides of (5) by $t^{\alpha-1}$, and integrating the resulting inequality with respect to $t$ over $[0,1]$, we obtain

$$
\int_{0}^{1} f\left(a^{t} b^{1-t}\right) t^{\alpha-1} d t+\int_{0}^{1} f\left(b^{t} a^{1-t}\right) t^{\alpha-1} d t \leq[f(a)+f(b)] \int_{0}^{1} t^{\alpha-1} d t
$$

i.e.,

$$
\frac{\Gamma(\alpha+1)}{\left(\ln \frac{b}{a}\right)^{\alpha}}\left\{J_{a+}^{\alpha} f(b)+J_{b-}^{\alpha} f(a)\right\} \leq f(a)+f(b) .
$$

The proof is completed.

In order to prove our main results, we need the following identity.

Lemma 2.1 Let $f: I \subseteq(0, \infty) \rightarrow \mathbb{R}$ be a differentiable function on $I^{\circ}$ such that $f^{\prime} \in L[a, b]$, where $a, b \in I$ with $a<b$. Then for all $x \in[a, b], \lambda \in[0,1]$ and $\alpha>0$, we have:

$$
\begin{aligned}
I_{f}(x, \lambda, \alpha, a, b)= & a\left(\ln \frac{x}{a}\right)^{\alpha+1} \int_{0}^{1}\left(t^{\alpha}-\lambda\right)\left(\frac{x}{a}\right)^{t} f^{\prime}\left(x^{t} a^{1-t}\right) d t \\
& -b\left(\ln \frac{b}{x}\right)^{\alpha+1} \int_{0}^{1}\left(t^{\alpha}-\lambda\right)\left(\frac{x}{b}\right)^{t} f^{\prime}\left(x^{t} b^{1-t}\right) d t .
\end{aligned}
$$

Proof By integration by parts and twice changing the variable, for $x \neq a$, we can state that

$$
\begin{aligned}
a & \ln \frac{x}{a} \int_{0}^{1}\left(t^{\alpha}-\lambda\right)\left(\frac{x}{a}\right)^{t} f^{\prime}\left(x^{t} a^{1-t}\right) d t \\
& =\int_{0}^{1}\left(t^{\alpha}-\lambda\right) d f\left(x^{t} a^{1-t}\right) \\
& =\left.\left(t^{\alpha}-\lambda\right) f\left(x^{t} a^{1-t}\right)\right|_{0} ^{1}-\frac{\alpha}{\left(\ln \frac{x}{a}\right)^{\alpha}} \int_{a}^{x}\left(\ln \frac{u}{a}\right)^{\alpha-1} \frac{f(u)}{u} d u \\
& =(1-\lambda) f(x)+\lambda f(a)-\frac{\Gamma(\alpha+1)}{\left(\ln \frac{x}{a}\right)^{\alpha}} J_{x-f}^{\alpha} f(a),
\end{aligned}
$$


and for $x \neq b$, similarly, we get

$$
\begin{aligned}
-b & \ln \frac{b}{x} \int_{0}^{1}\left(t^{\alpha}-\lambda\right)\left(\frac{x}{b}\right)^{t} f^{\prime}\left(x^{t} b^{1-t}\right) d t \\
= & \int_{0}^{1}\left(t^{\alpha}-\lambda\right) d f\left(x^{t} b^{1-t}\right) \\
= & \left.\left(t^{\alpha}-\lambda\right) f\left(x^{t} b^{1-t}\right)\right|_{0} ^{1}-\frac{\alpha}{\left(\ln \frac{b}{x}\right)^{\alpha}} \int_{x}^{b}\left(\ln \frac{b}{u}\right)^{\alpha-1} \frac{f(u)}{u} d u \\
= & (1-\lambda) f(x)+\lambda f(b)-\frac{\Gamma(\alpha+1)}{\left(\ln \frac{b}{x}\right)^{\alpha}} J_{x+1}^{\alpha} f(b) .
\end{aligned}
$$

Multiplying both sides of (7) and (8) by $\left(\ln \frac{x}{a}\right)^{\alpha}$ and $\left(\ln \frac{b}{x}\right)^{\alpha}$, respectively, and adding the resulting identities, we obtain the desired result. For $x=a$ and $x=b$, the identities

$$
I_{f}(a, \lambda, \alpha ; a, b)=b\left(\ln \frac{b}{a}\right)^{\alpha+1} \int_{0}^{1}\left(t^{\alpha}-\lambda\right)\left(\frac{a}{b}\right)^{t} f^{\prime}\left(a^{t} b^{1-t}\right) d t
$$

and

$$
I_{f}(b, \lambda, \alpha ; a, b)=a\left(\ln \frac{b}{a}\right)^{\alpha+1} \int_{0}^{1}\left(t^{\alpha}-\lambda\right)\left(\frac{b}{a}\right)^{t} f^{\prime}\left(b^{t} a^{1-t}\right),
$$

can be proved respectively easily by performing an integration by parts in the integrals from the right-hand side and changing the variable.

Theorem 2.2 Let $f: I \subset(0, \infty) \rightarrow \mathbb{R}$ be a differentiable function on $I^{\circ}$ such that $f^{\prime} \in$ $L[a, b]$, where $a, b \in I^{\circ}$ with $a<b$. If $\left|f^{\prime}\right|^{q}$ is quasi-geometrically convex on $[a, b]$ for some fixed $q \geq 1, x \in[a, b], \lambda \in[0,1]$ and $\alpha>0$, then the following inequality for fractional integrals holds:

$$
\begin{aligned}
& \left|I_{f}(x, \lambda, \alpha, a, b)\right| \\
& \quad \leq A_{1}^{1-\frac{1}{q}}(\alpha, \lambda)\left\{a\left(\ln \frac{x}{a}\right)^{\alpha+1}\left(\sup \left\{\left|f^{\prime}(x)\right|^{q},\left|f^{\prime}(a)\right|^{q}\right\}\right)^{\frac{1}{q}} B_{1}^{\frac{1}{q}}(x, \alpha, \lambda, q)\right. \\
& \left.\quad+b\left(\ln \frac{b}{x}\right)^{\alpha+1}\left(\sup \left\{\left|f^{\prime}(x)\right|^{q},\left|f^{\prime}(b)\right|^{q}\right\}\right)^{\frac{1}{q}} B_{2}^{\frac{1}{q}}(x, \alpha, \lambda, q)\right\},
\end{aligned}
$$

where

$$
\begin{aligned}
& A_{1}(\alpha, \lambda)=\frac{2 \alpha \lambda^{1+\frac{1}{\alpha}}+1}{\alpha+1}-\lambda, \\
& B_{1}(x, \alpha, \lambda, q)=\int_{0}^{1}\left|t^{\alpha}-\lambda\right|\left(\frac{x}{a}\right)^{q t} d t, \\
& B_{2}(x, \alpha, \lambda, q)=\int_{0}^{1}\left|t^{\alpha}-\lambda\right|\left(\frac{x}{b}\right)^{q t} d t .
\end{aligned}
$$

Proof Since $\left|f^{\prime}\right|^{q}$ is quasi-geometrically convex on $[a, b]$, for all $t \in[0,1]$,

$$
\left|f^{\prime}\left(x^{t} a^{1-t}\right)\right|^{q} \leq \sup \left\{\left|f^{\prime}(x)\right|^{q},\left|f^{\prime}(a)\right|^{q}\right\}
$$


and

$$
\left|f^{\prime}\left(x^{t} b^{1-t}\right)\right|^{q} \leq \sup \left\{\left|f^{\prime}(x)\right|^{q},\left|f^{\prime}(b)\right|^{q}\right\}
$$

Hence, using Lemma 2.1 and power mean inequality, we get

$$
\begin{aligned}
& \left|I_{f}(x, \lambda, \alpha, a, b)\right| \\
& \leq a\left(\ln \frac{x}{a}\right)^{\alpha+1}\left(\int_{0}^{1}\left|t^{\alpha}-\lambda\right| d t\right)^{1-\frac{1}{q}}\left(\int_{0}^{1}\left|t^{\alpha}-\lambda\right|\left(\frac{x}{a}\right)^{q t} \sup \left\{\left|f^{\prime}(x)\right|^{q},\left|f^{\prime}(a)\right|^{q}\right\} d t\right)^{\frac{1}{q}} \\
& +b\left(\ln \frac{b}{x}\right)^{\alpha+1}\left(\int_{0}^{1}\left|t^{\alpha}-\lambda\right| d t\right)^{1-\frac{1}{q}}\left(\int_{0}^{1}\left|t^{\alpha}-\lambda\right|\left(\frac{x}{b}\right)^{q t} \sup \left\{\left|f^{\prime}(x)\right|^{q},\left|f^{\prime}(b)\right|^{q}\right\} d t\right)^{\frac{1}{q}}, \\
& \left|I_{f}(x, \lambda, \alpha, a, b)\right| \leq\left(\int_{0}^{1}\left|t^{\alpha}-\lambda\right| d t\right)^{1-\frac{1}{q}} \\
& \times\left\{a\left(\ln \frac{x}{a}\right)^{\alpha+1}\left(\sup \left\{\left|f^{\prime}(x)\right|^{q},\left|f^{\prime}(a)\right|^{q}\right\}\right)^{\frac{1}{q}}\left(\int_{0}^{1}\left|t^{\alpha}-\lambda\right|\left(\frac{x}{a}\right)^{q t} d t\right)^{\frac{1}{q}}\right. \\
& \left.+b\left(\ln \frac{b}{x}\right)^{\alpha+1}\left(\sup \left\{\left|f^{\prime}(x)\right|^{q},\left|f^{\prime}(b)\right|^{q}\right\}\right)^{\frac{1}{q}}\left(\int_{0}^{1}\left|t^{\alpha}-\lambda\right|\left(\frac{x}{b}\right)^{q t} d t\right)^{\frac{1}{q}}\right\} \\
& \leq A_{1}^{1-\frac{1}{q}}(\alpha, \lambda)\left\{a\left(\ln \frac{x}{a}\right)^{\alpha+1}\left(\sup \left\{\left|f^{\prime}(x)\right|^{q},\left|f^{\prime}(a)\right|^{q}\right\}\right)^{\frac{1}{q}} B_{1}^{\frac{1}{q}}(x, \alpha, \lambda, q)\right. \\
& \left.+b\left(\ln \frac{b}{x}\right)^{\alpha+1}\left(\sup \left\{\left|f^{\prime}(x)\right|^{q},\left|f^{\prime}(b)\right|^{q}\right\}\right)^{\frac{1}{q}} B_{2}^{\frac{1}{q}}(x, \alpha, \lambda, q)\right\},
\end{aligned}
$$

which completes the proof.

Corollary 2.1 Under the assumptions of Theorem 2.2 with $q=1$, inequality (9) reduces to the following inequality:

$$
\begin{aligned}
\left|I_{f}(x, \lambda, \alpha, a, b)\right| \leq & \left\{a\left(\ln \frac{x}{a}\right)^{\alpha+1} B_{1}(x, \alpha, \lambda, 1) \sup \left\{\left|f^{\prime}(x)\right|,\left|f^{\prime}(a)\right|\right\}\right. \\
& \left.+b\left(\ln \frac{b}{x}\right)^{\alpha+1} B_{2}(x, \alpha, \lambda, 1) \sup \left\{\left|f^{\prime}(x)\right|,\left|f^{\prime}(b)\right|\right\}\right\} .
\end{aligned}
$$

Corollary 2.2 Under the assumptions of Theorem 2.2 with $\alpha=1$, inequality (9) reduces to the following inequality:

$$
\begin{aligned}
& \left(\ln \frac{b}{a}\right)^{-1}\left|I_{f}(x, \lambda, \alpha, a, b)\right| \\
& \leq\left|(1-\lambda) f(x)+\lambda\left[\frac{f(a) \ln \frac{x}{a}+f(b) \ln \frac{b}{x}}{\ln \frac{b}{a}}\right]-\frac{1}{\ln \frac{b}{a}} \int_{a}^{b} \frac{f(u)}{u} d u\right| \\
& \leq\left(\ln \frac{b}{a}\right)^{-1}\left(\frac{2 \lambda^{2}-2 \lambda+1}{2}\right)^{1-\frac{1}{q}}\left\{a\left(\ln \frac{x}{a}\right)^{2} B_{1}^{\frac{1}{q}}(x, 1, \lambda, q)\left(\sup \left\{\left|f^{\prime}(x)\right|^{q},\left|f^{\prime}(a)\right|^{q}\right\}\right)^{\frac{1}{q}}\right. \\
& \left.\quad+b\left(\ln \frac{b}{x}\right)^{2} B_{2}^{\frac{1}{q}}(x, 1, \lambda, q)\left(\sup \left\{\left|f^{\prime}(x)\right|^{q},\left|f^{\prime}(b)\right|^{q}\right\}\right)^{\frac{1}{q}}\right\}
\end{aligned}
$$


where

$$
\begin{aligned}
& B_{1}(x, 1, \lambda, q)=h_{\lambda}\left(\left(\frac{x}{a}\right)^{q}\right), \quad B_{2}(x, 1, \lambda, q)=h_{\lambda}\left(\left(\frac{x}{b}\right)^{q}\right), \\
& h(u, \lambda)=\frac{2 u^{\lambda}-u-1}{(\ln u)^{2}}+\frac{(1-\lambda) u-\lambda}{\ln u}, \quad u \in(0, \infty) \backslash\{1\},
\end{aligned}
$$

specially for $x=\sqrt{a b}$, we get

$$
\begin{aligned}
\mid(1 & -\lambda) f(\sqrt{a b})+\lambda\left(\frac{f(a)+f(b)}{2}\right)-\frac{1}{\ln \frac{b}{a}} \int_{a}^{b} \frac{f(u)}{u} d u \mid \\
\leq & \frac{\ln \frac{b}{a}}{4}\left(\frac{2 \lambda^{2}-2 \lambda+1}{2}\right)^{1-\frac{1}{q}}\left\{a h^{\frac{1}{q}}\left(\left(\frac{b}{a}\right)^{\frac{q}{2}}, \lambda\right)\left(\sup \left\{\left|f^{\prime}(\sqrt{a b})\right|^{q},\left|f^{\prime}(a)\right|^{q}\right\}\right)^{\frac{1}{q}}\right. \\
& \left.+b h^{\frac{1}{q}}\left(\left(\frac{a}{b}\right)^{\frac{q}{2}}, \lambda\right)\left(\sup \left\{\left|f^{\prime}(\sqrt{a b})\right|^{q},\left|f^{\prime}(b)\right|^{q}\right\}\right)^{\frac{1}{q}}\right\} .
\end{aligned}
$$

\section{Corollary 2.3 In Theorem 2.2,}

1. If we take $x=\sqrt{a b}, \lambda=\frac{1}{3}$, then we get the following Simpson-type inequality for fractional integrals:

$$
\begin{aligned}
\mid \frac{1}{6}[ & f(a)+4 f(\sqrt{a b})+f(b)]-\frac{2^{\alpha-1} \Gamma(\alpha+1)}{\left(\ln \frac{b}{a}\right)^{\alpha}}\left[J_{\sqrt{a b}}^{\alpha} f(a)+J_{\sqrt{a b}+}^{\alpha} f(b)\right] \mid \\
\leq & \frac{\ln \frac{b}{a}}{4} A_{1}^{1-\frac{1}{q}}\left(\alpha, \frac{1}{3}\right)\left\{a\left[\sup \left\{\left|f^{\prime}(\sqrt{a b})\right|^{q},\left|f^{\prime}(a)\right|^{q}\right\}\right]^{\frac{1}{q}} B_{1}^{\frac{1}{q}}\left(\sqrt{a b}, \alpha, \frac{1}{3}, q\right)\right. \\
& \left.+b\left[\sup \left\{\left|f^{\prime}(\sqrt{a b})\right|^{q},\left|f^{\prime}(b)\right|^{q}\right\}\right]^{\frac{1}{q}} B_{2}^{\frac{1}{q}}\left(\sqrt{a b}, \alpha, \frac{1}{3}, q\right)\right\},
\end{aligned}
$$

specially for $\alpha=1$, we get

$$
\begin{aligned}
& \left|\frac{1}{6}[f(a)+4 f(\sqrt{a b})+f(b)]-\frac{1}{\ln \frac{b}{a}} \int_{a}^{b} \frac{f(u)}{u} d u\right| \\
& \leq \frac{\ln \frac{b}{a}}{4}\left(\frac{5}{18}\right)^{1-\frac{1}{q}}\left\{a\left[\sup \left\{\left|f^{\prime}(\sqrt{a b})\right|,\left|f^{\prime}(a)\right|\right\}\right]^{\frac{1}{q}} h^{\frac{1}{q}}\left(\left(\frac{b}{a}\right)^{\frac{q}{2}}, \frac{1}{3}\right)\right. \\
& \left.+b\left[\sup \left\{\left|f^{\prime}(\sqrt{a b})\right|^{q},\left|f^{\prime}(b)\right|^{q}\right\}\right]^{\frac{1}{q}} h^{\frac{1}{q}}\left(\left(\frac{a}{b}\right)^{\frac{q}{2}}, \frac{1}{3}\right)\right\},
\end{aligned}
$$

where $h$ is defined as in (10).

\section{Remark 2.2}

1. If we take $x=\sqrt{a b}, \lambda=0$, then we get the following midpoint- type inequality for fractional integrals:

$$
\begin{aligned}
& \left|f(\sqrt{a b})-\frac{2^{\alpha-1} \Gamma(\alpha+1)}{\left(\ln \frac{b}{a}\right)^{\alpha}}\left[J_{\sqrt{a b}}^{\alpha} f(a)+J_{\sqrt{a b+}}^{\alpha} f(b)\right]\right| \\
& \leq \frac{\ln \frac{b}{a}}{4}\left(\frac{1}{\alpha+1}\right)^{1-\frac{1}{q}}\left\{a\left[\sup \left\{\left|f^{\prime}(\sqrt{a b})\right|^{q},\left|f^{\prime}(a)\right|^{q}\right\}\right]^{\frac{1}{q}} B_{1}^{\frac{1}{q}}(\sqrt{a b}, 1,0, q)\right. \\
& \left.\quad+b\left[\sup \left\{\left|f^{\prime}(\sqrt{a b})\right|^{q},\left|f^{\prime}(b)\right|^{q}\right\}\right]^{\frac{1}{q}} B_{2}^{\frac{1}{q}}(\sqrt{a b}, 1,0, q)\right\},
\end{aligned}
$$


specially for $\alpha=1$, we get

$$
\begin{aligned}
& \left|f(\sqrt{a b})-\frac{1}{\ln \frac{b}{a}} \int_{a}^{b} \frac{f(u)}{u} d u\right| \\
& \leq \frac{2^{\frac{1}{q}} \ln \frac{b}{a}}{8}\left\{a\left[\sup \left\{\left|f^{\prime}(\sqrt{a b})\right|^{q},\left|f^{\prime}(a)\right|^{q}\right\}\right]^{\frac{1}{q}} h^{\frac{1}{q}}\left(\left(\frac{b}{a}\right)^{\frac{q}{2}}, 0\right)\right. \\
& \left.\quad+b\left[\sup \left\{\left|f^{\prime}(\sqrt{a b})\right|^{q},\left|f^{\prime}(b)\right|^{q}\right\}\right]^{\frac{1}{q}} h^{\frac{1}{q}}\left(\left(\frac{a}{b}\right)^{\frac{q}{2}}, 0\right)\right\},
\end{aligned}
$$

where $h$ is defined as in (10).

2. If we take $x=\sqrt{a b}, \lambda=1$, then we get the following trapezoid-type inequality for fractional integrals:

$$
\begin{aligned}
& \left|\frac{f(a)+f(b)}{2}-\frac{2^{\alpha-1} \Gamma(\alpha+1)}{\left(\ln \frac{b}{a}\right)^{\alpha}}\left[J_{\sqrt{a b}-}^{\alpha} f(a)+J_{\sqrt{a b}+}^{\alpha} f(b)\right]\right| \\
& \leq \frac{\ln \frac{b}{a}}{4}\left(\frac{1}{\alpha+1}\right)^{1-\frac{1}{q}}\left\{a\left[\sup \left\{\left|f^{\prime}(\sqrt{a b})\right|^{q},\left|f^{\prime}(a)\right|^{q}\right\}\right]^{\frac{1}{q}} B_{1}^{\frac{1}{q}}(\sqrt{a b}, \alpha, 1, q)\right. \\
& \left.\quad+b\left[\sup \left\{\left|f^{\prime}(\sqrt{a b})\right|^{q},\left|f^{\prime}(b)\right|^{q}\right\}\right]^{\frac{1}{q}} B_{2}^{\frac{1}{q}}(\sqrt{a b}, \alpha, 1, q)\right\},
\end{aligned}
$$

specially for $\alpha=1$, we get

$$
\begin{aligned}
& \left|\frac{f(a)+f(b)}{2}-\frac{1}{\ln \frac{b}{a}} \int_{a}^{b} \frac{f(u)}{u} d u\right| \\
& \leq \frac{2^{\frac{1}{q}} \ln \frac{b}{a}}{8}\left\{a\left[\sup \left\{\left|f^{\prime}(\sqrt{a b})\right|^{q},\left|f^{\prime}(a)\right|^{q}\right\}\right]^{\frac{1}{q}} h^{\frac{1}{q}}\left(\left(\frac{b}{a}\right)^{\frac{q}{2}}, 1\right)\right. \\
& \left.\quad+b\left[\sup \left\{\left|f^{\prime}(\sqrt{a b})\right|^{q},\left|f^{\prime}(b)\right|^{q}\right\}\right]^{\frac{1}{q}} h^{\frac{1}{q}}\left(\left(\frac{a}{b}\right)^{\frac{q}{2}}, 1\right)\right\},
\end{aligned}
$$

where $h$ is defined as in (10).

Corollary 2.4 Let the assumptions of Theorem 2.2 hold. If $\left|f^{\prime}(x)\right| \leq M$ for all $x \in[a, b]$ and $\lambda=0$, then we get the following Ostrowski-type inequality for fractional integrals from inequality (9):

$$
\begin{aligned}
& \left|\left[\left(\ln \frac{x}{a}\right)^{\alpha}+\left(\ln \frac{b}{x}\right)^{\alpha}\right] f(x)-\Gamma(\alpha+1)\left[J_{\sqrt{a b}}^{\alpha} f(a)+J_{\sqrt{a b}+}^{\alpha} f(b)\right]\right| \\
& \quad \leq \frac{M}{(\alpha+1)^{1-\frac{1}{q}}}\left[a\left(\ln \frac{x}{a}\right)^{\alpha+1} B_{1}^{\frac{1}{q}}(x, \alpha, 0, q)+b\left(\ln \frac{b}{x}\right)^{\alpha+1} B_{2}^{\frac{1}{q}}(x, \alpha, 0, q)\right] .
\end{aligned}
$$

Theorem 2.3 Let $f: I \subset(0, \infty) \rightarrow \mathbb{R}$ be a differentiable function on $I^{\circ}$ such that $f^{\prime} \in$ $L[a, b]$, where $a, b \in I^{\circ}$ with $a<b$. If $\left|f^{\prime}\right|^{q}$ is quasi-geometrically convex on $[a, b]$ for some fixed $q>1, x \in[a, b], \lambda \in[0,1]$ and $\alpha>0$, then the following inequality for fractional inte- 
grals holds:

$$
\begin{aligned}
& \left|I_{f}(x, \lambda, \alpha, a, b)\right| \\
& \quad \leq A_{2}^{\frac{1}{p}}(\alpha, \lambda, p)\left\{a\left(\ln \frac{x}{a}\right)^{\alpha+\frac{1}{p}}\left(\sup \left\{\left|f^{\prime}(x)\right|^{q},\left|f^{\prime}(a)\right|^{q}\right\}\right)^{\frac{1}{q}}\left(\frac{x^{q}-a^{q}}{q}\right)^{\frac{1}{q}}\right. \\
& \left.\quad+b\left(\ln \frac{b}{x}\right)^{\alpha+\frac{1}{p}}\left(\sup \left\{\left|f^{\prime}(x)\right|^{q},\left|f^{\prime}(b)\right|^{q}\right\}\right)^{\frac{1}{q}}\left(\frac{b^{q}-x^{q}}{q}\right)^{\frac{1}{q}}\right\},
\end{aligned}
$$

where

$$
\begin{aligned}
& A_{2}(\alpha, \lambda, p) \\
& \quad= \begin{cases}\frac{1}{\alpha p+1}, & \lambda=0, \\
\frac{\lambda\left(\frac{\alpha p+1}{\alpha}\right.}{\alpha}\left\{\beta\left(\frac{1}{\alpha}, p+1\right)+\frac{(1-\lambda)^{p+1}}{p+1}\right. & \\
\left.\quad \times{ }_{2} F_{1}\left(\frac{1}{\alpha}+p+1, p+1, p+2 ; 1-\lambda\right)\right\}, & 0<\lambda<1, \\
\frac{1}{\alpha} \beta\left(p+1, \frac{1}{\alpha}\right), & \lambda=1,\end{cases}
\end{aligned}
$$

${ }_{2} F_{1}$ is hypergeometric function defined by

$$
{ }_{2} F_{1}(a, b ; c ; z)=\frac{1}{\beta(b, c-b)} \int_{0}^{1} t^{b-1}(1-t)^{c-b-1}(1-z t)^{-a} d t, \quad c>b>0,|z|<1(\text { see }[21]),
$$

and $\frac{1}{p}+\frac{1}{q}=1$.

Proof Using Lemma 2.1, the Hölder inequality and quasi-geometrical convexity of $\left|f^{\prime}\right|^{q}$, we get

$$
\begin{aligned}
&\left|I_{f}(x, \lambda, \alpha, a, b)\right|=a\left(\ln \frac{x}{a}\right)^{\alpha+1}\left(\int_{0}^{1}\left|t^{\alpha}-\lambda\right|^{p} d t\right)^{\frac{1}{p}}\left(\int_{0}^{1}\left(\frac{x}{a}\right)^{q t} \sup \left\{\left|f^{\prime}(x)\right|^{q},\left|f^{\prime}(a)\right|^{q}\right\} d t\right)^{\frac{1}{q}} \\
&+b\left(\ln \frac{b}{x}\right)^{\alpha+1}\left(\int_{0}^{1}\left|t^{\alpha}-\lambda\right|^{p} d t\right)^{\frac{1}{p}}\left(\int_{0}^{1}\left(\frac{x}{b}\right)^{q t} \sup \left\{\left|f^{\prime}(x)\right|^{q},\left|f^{\prime}(b)\right|^{q}\right\} d t\right)^{\frac{1}{q}}, \\
&\left|I_{f}(x, \lambda, \alpha, a, b)\right| \leq\left(\int_{0}^{1}\left|t^{\alpha}-\lambda\right|^{p} d t\right)^{\frac{1}{p}} \\
& \times\left\{a\left(\ln \frac{x}{a}\right)^{\alpha+1}\left(\sup \left\{\left|f^{\prime}(x)\right|^{q},\left|f^{\prime}(a)\right|^{q}\right\}\right)^{\frac{1}{q}}\left(\int_{0}^{1}\left(\frac{x}{a}\right)^{q t} d t\right)^{\frac{1}{q}}\right. \\
&\left.+b\left(\ln \frac{b}{x}\right)^{\alpha+1}\left(\sup \left\{\left|f^{\prime}(x)\right|^{q},\left|f^{\prime}(b)\right|^{q}\right\}\right)^{\frac{1}{q}}\left(\int_{0}^{1}\left(\frac{x}{b}\right)^{q t} d t\right)^{\frac{1}{q}}\right\} \\
& \leq A_{2}^{\frac{1}{p}}(\alpha, \lambda, p)\left\{a\left(\ln \frac{x}{a}\right)^{\alpha+1-\frac{1}{q}}\left(\sup \left\{\left|f^{\prime}(x)\right|^{q},\left|f^{\prime}(a)\right|^{q}\right\}\right)^{\frac{1}{q}}\left(\frac{x^{q}-a^{q}}{q}\right)^{\frac{1}{q}}\right. \\
&\left.+b\left(\ln \frac{b}{x}\right)^{\alpha+1-\frac{1}{q}}\left(\sup \left\{\left|f^{\prime}(x)\right|^{q},\left|f^{\prime}(b)\right|^{q}\right\}\right)^{\frac{1}{q}}\left(\frac{b^{q}-x^{q}}{q}\right)^{\frac{1}{q}}\right\},
\end{aligned}
$$


here, it is seen by a simple computation that

$$
\begin{aligned}
A_{2}(\alpha, \lambda, p) & =\int_{0}^{1}\left|t^{\alpha}-\lambda\right|^{p} d t \\
& = \begin{cases}\frac{1}{\alpha p+1}, & \lambda=0, \\
\frac{\lambda^{\frac{\alpha p+1}{\alpha}}}{\alpha}\left\{\beta\left(\frac{1}{\alpha}, p+1\right)+\frac{(1-\lambda)^{p+1}}{p+1}\right. & \\
\left.\times{ }_{2} F_{1}\left(\frac{1}{\alpha}+p+1, p+1,2+p ; 1-\lambda\right)\right\}, & 0<\lambda<1, \\
\frac{1}{\alpha} \beta\left(p+1, \frac{1}{\alpha}\right), & \lambda=1 .\end{cases}
\end{aligned}
$$

Hence, the proof is completed.

Corollary 2.5 Under the assumptions of Theorem 2.3 with $\alpha=1$, inequality (12) reduces to the following inequality:

$$
\begin{aligned}
\mid(1- & \lambda) f(x)+\lambda\left[\frac{f(a) \ln \frac{x}{a}+f(b) \ln \frac{b}{x}}{\ln \frac{b}{a}}\right]-\frac{1}{\ln \frac{b}{a}} \int_{a}^{b} \frac{f(u)}{u} d u \mid \\
\leq & \left(\ln \frac{b}{a}\right)^{-1}\left(\frac{\lambda^{p+1}+(1-\lambda)^{p+1}}{p+1}\right)^{\frac{1}{p}} \\
& \times\left\{a\left(\ln \frac{x}{a}\right)^{1+\frac{1}{p}}\left(\sup \left\{\left|f^{\prime}(x)\right|^{q},\left|f^{\prime}(a)\right|^{q}\right\}\right)^{\frac{1}{q}}\left(\frac{x^{q}-a^{q}}{q}\right)^{\frac{1}{q}}\right. \\
& \left.+b\left(\ln \frac{b}{x}\right)^{1+\frac{1}{p}}\left(\sup \left\{\left|f^{\prime}(x)\right|^{q},\left|f^{\prime}(b)\right|^{q}\right\}\right)^{\frac{1}{q}}\left(\frac{b^{q}-x^{q}}{q}\right)^{\frac{1}{q}}\right\}
\end{aligned}
$$

specially for $x=\sqrt{a b}$, we get

$$
\begin{aligned}
\mid(1- & \lambda) f(\sqrt{a b})+\lambda\left(\frac{f(a)+f(b)}{2}\right)-\frac{1}{\ln \frac{b}{a}} \int_{a}^{b} \frac{f(u)}{u} d u \mid \\
\leq & \frac{1}{2}\left(\frac{\ln \frac{b}{a}\left(\lambda^{p+1}+(1-\lambda)^{p+1}\right)}{2(p+1)}\right)^{\frac{1}{p}}\left\{a\left(\sup \left\{\left|f^{\prime}(\sqrt{a b})\right|^{q},\left|f^{\prime}(a)\right|^{q}\right\}\right)^{\frac{1}{q}}\left(\frac{\sqrt{a b}{ }^{q}-a^{q}}{q}\right)^{\frac{1}{q}}\right. \\
& \left.+b\left(\sup \left\{\left|f^{\prime}(\sqrt{a b})\right|^{q},\left|f^{\prime}(b)\right|^{q}\right\}\right)^{\frac{1}{q}}\left(\frac{b^{q}-\sqrt{a b}^{q}}{q}\right)^{\frac{1}{q}}\right\} .
\end{aligned}
$$

\section{Corollary 2.6 In Theorem 2.3,}

1. If we take $x=\sqrt{a b}, \lambda=\frac{1}{3}$, then we get the following Simpson-type inequality for fractional integrals:

$$
\begin{aligned}
& \left|\frac{1}{6}[f(a)+4 f(\sqrt{a b})+f(b)]-\frac{2^{\alpha-1} \Gamma(\alpha+1)}{\left(\ln \frac{b}{a}\right)^{\alpha}}\left[J_{\sqrt{a b}-}^{\alpha} f(a)+J_{\sqrt{a b}+}^{\alpha} f(b)\right]\right| \\
& \leq \frac{1}{2}\left(\frac{\ln \frac{b}{a}\left(1+2^{p+1}\right)}{3^{p+1}(p+1) 2}\right)^{\frac{1}{p}}\left\{a\left[\sup \left\{\left|f^{\prime}(\sqrt{a b})\right|^{q},\left|f^{\prime}(a)\right|^{q}\right\}\right]^{\frac{1}{q}}\left(\frac{\sqrt{a b}-a^{q}}{q}\right)^{\frac{1}{q}}\right. \\
& \left.\quad+b\left[\sup \left\{\left|f^{\prime}(\sqrt{a b})\right|^{q},\left|f^{\prime}(b)\right|^{q}\right\}\right]^{\frac{1}{q}}\left(\frac{b^{q}-\sqrt{a b}^{q}}{q}\right)^{\frac{1}{q}}\right\}
\end{aligned}
$$


specially for $\alpha=1$, we get

$$
\begin{aligned}
\left|\frac{1}{6}[f(a)+4 f(\sqrt{a b})+f(b)]-\frac{1}{\ln \frac{b}{a}} \int_{a}^{b} \frac{f(u)}{u} d u\right| \\
\leq \frac{1}{2}\left(\frac{\ln \frac{b}{a}\left(1+2^{p+1}\right)}{3^{p+1}(p+1) 2}\right)^{\frac{1}{p}} \\
\quad \times\left\{a\left[\sup \left\{\left|f^{\prime}(\sqrt{a b})\right|^{q},\left|f^{\prime}(a)\right|^{q}\right\}\right]^{\frac{1}{q}}\left(\frac{\sqrt{a b}^{q}-a^{q}}{q}\right)^{\frac{1}{q}}\right. \\
\left.\quad+b\left[\sup \left\{\left|f^{\prime}(\sqrt{a b})\right|^{q},\left|f^{\prime}(b)\right|^{q}\right\}\right]^{\frac{1}{q}}\left(\frac{b^{q}-\sqrt{a b}^{q}}{q}\right)^{\frac{1}{q}}\right\} .
\end{aligned}
$$

\section{Remark 2.3}

1. If we take $x=\sqrt{a b}, \lambda=0$, then we get the following midpoint- type inequality for fractional integrals:

$$
\begin{aligned}
& \left|f(\sqrt{a b})-\frac{2^{\alpha-1} \Gamma(\alpha+1)}{\left(\ln \frac{b}{a}\right)^{\alpha}}\left[J_{\sqrt{a b}}^{\alpha} f(a)+J_{\sqrt{a b}+}^{\alpha} f(b)\right]\right| \\
& \leq \frac{1}{2}\left(\frac{\ln \frac{b}{a}}{2(\alpha p+1)}\right)^{\frac{1}{p}}\left\{a\left[\sup \left\{\left|f^{\prime}(\sqrt{a b})\right|^{q},\left|f^{\prime}(a)\right|^{q}\right\}\right]^{\frac{1}{q}}\left(\frac{\sqrt{a b}^{q}-a^{q}}{q}\right)^{\frac{1}{q}}\right. \\
& \left.\quad+b\left[\sup \left\{\left|f^{\prime}(\sqrt{a b})\right|^{q},\left|f^{\prime}(b)\right|^{q}\right\}\right]^{\frac{1}{q}}\left(\frac{b^{q}-\sqrt{a b}^{q}}{q}\right)^{\frac{1}{q}}\right\}
\end{aligned}
$$

specially for $\alpha=1$, we get

$$
\begin{aligned}
\mid f(\sqrt{a b}) & -\frac{1}{\ln \frac{b}{a}} \int_{a}^{b} \frac{f(u)}{u} d u \mid \\
\leq & \frac{1}{2}\left(\frac{\ln \frac{b}{a}}{2(p+1)}\right)^{\frac{1}{p}}\left\{a\left[\sup \left\{\left|f^{\prime}(\sqrt{a b})\right|^{q},\left|f^{\prime}(a)\right|^{q}\right\}\right]^{\frac{1}{q}}\left(\frac{\sqrt{a b}-a^{q}}{q}\right)^{\frac{1}{q}}\right. \\
& \left.+b\left[\sup \left\{\left|f^{\prime}(\sqrt{a b})\right|^{q},\left|f^{\prime}(b)\right|^{q}\right\}\right]^{\frac{1}{q}}\left(\frac{b^{q}-\sqrt{a b}}{q}\right)^{q}\right\} .
\end{aligned}
$$

2. If we take $x=\sqrt{a b}, \lambda=1$, then we get the following trapezoid-type inequality for fractional integrals $\frac{1}{\alpha} \beta\left(p+1, \frac{1}{\alpha}\right)$ :

$$
\begin{aligned}
& \left|\frac{f(a)+f(b)}{2}-\frac{2^{\alpha-1} \Gamma(\alpha+1)}{\left(\ln \frac{b}{a}\right)^{\alpha}}\left[J_{\sqrt{a b}}^{\alpha} f(a)+J_{\sqrt{a b}+}^{\alpha} f(b)\right]\right| \\
& \leq \frac{1}{2}\left(\frac{\ln \frac{b}{a} \beta\left(p+1, \frac{1}{\alpha}\right)}{2 \alpha}\right)^{\frac{1}{p}} \\
& \quad \times\left\{a\left[\sup \left\{\left|f^{\prime}(\sqrt{a b})\right|^{q},\left|f^{\prime}(a)\right|^{q}\right\}\right]^{\frac{1}{q}}\left(\frac{\sqrt{a b}-a^{q}}{q}\right)^{\frac{1}{q}}\right. \\
& \left.+b\left[\sup \left\{\left|f^{\prime}(\sqrt{a b})\right|^{q},\left|f^{\prime}(b)\right|^{q}\right\}\right]^{\frac{1}{q}}\left(\frac{b^{q}-\sqrt{a b}^{q}}{q}\right)^{\frac{1}{q}}\right\},
\end{aligned}
$$


specially for $\alpha=1$, we get

$$
\begin{aligned}
& \left|\frac{f(a)+f(b)}{2}-\frac{1}{\ln \frac{b}{a}} \int_{a}^{b} \frac{f(u)}{u} d u\right| \\
& \leq \frac{1}{2}\left(\frac{\ln \frac{b}{a}}{2(p+1)}\right)^{\frac{1}{p}}\left\{a\left[\sup \left\{\left|f^{\prime}(\sqrt{a b})\right|^{q},\left|f^{\prime}(a)\right|^{q}\right\}\right]^{\frac{1}{q}}\left(\frac{\sqrt{a b^{q}}-a^{q}}{q}\right)^{\frac{1}{q}}\right. \\
& \left.\quad+b\left[\sup \left\{\left|f^{\prime}(\sqrt{a b})\right|^{q},\left|f^{\prime}(b)\right|^{q}\right\}\right]^{\frac{1}{q}}\left(\frac{b^{q}-\sqrt{a b^{q}}}{q}\right)^{\frac{1}{q}}\right\} .
\end{aligned}
$$

Corollary 2.7 Let the assumptions of Theorem 2.3 hold. If $\left|f^{\prime}(x)\right| \leq M$ for all $x \in[a, b]$ and $\lambda=0$, then we get the following Ostrowski-type inequality for fractional integrals from inequality (12):

$$
\begin{aligned}
& \left|\left[\left(\ln \frac{x}{a}\right)^{\alpha}+\left(\ln \frac{b}{x}\right)^{\alpha}\right] f(x)-\Gamma(\alpha+1)\left[J_{\sqrt{a b}}^{\alpha} f(a)+J_{\sqrt{a b}+}^{\alpha} f(b)\right]\right| \\
& \quad \leq \frac{M}{(\alpha p+1)^{\frac{1}{p}}}\left[a\left(\ln \frac{x}{a}\right)^{\alpha+\frac{1}{p}}\left(\frac{x^{q}-a^{q}}{q}\right)^{\frac{1}{q}}+b\left(\ln \frac{b}{x}\right)^{\alpha+\frac{1}{p}}\left(\frac{b^{q}-x^{q}}{q}\right)^{\frac{1}{q}}\right] .
\end{aligned}
$$

\section{Application to special means}

Let us recall the following special means of two nonnegative numbers $a, b$ with $b>a$ :

1. The arithmetic mean

$$
A=A(a, b):=\frac{a+b}{2} .
$$

2. The geometric mean

$$
G=G(a, b):=\sqrt{a b}
$$

3. The logarithmic mean

$$
L=L(a, b):=\frac{b-a}{\ln b-\ln a} .
$$

4. The $p$-logarithmic mean

$$
L_{p}=L_{p}(a, b):=\left(\frac{b^{p+1}-a^{p+1}}{(p+1)(b-a)}\right)^{\frac{1}{p}}, \quad p \in \mathbb{R} \backslash\{-1,0\} .
$$

Proposition 3.1 For $b>a>0, n>0$ and $q \geq 1$, we have

$$
\begin{aligned}
& \left|(1-\lambda) G^{n+1}(a, b)+\lambda A\left(a^{n+1}, b^{n+1}\right)-(n+1) L(a, b) L_{n}^{n}(a, b)\right| \\
& \quad \leq \frac{(n+1) \ln \frac{b}{a}}{4}\left(\frac{2 \lambda^{2}-2 \lambda+1}{2}\right)^{1-\frac{1}{q}}\left\{a G^{n}(a, b) h^{\frac{1}{q}}\left(\left(\frac{b}{a}\right)^{\frac{q}{2}}, \lambda\right)\right. \\
& \left.\quad+b^{n+1} h^{\frac{1}{q}}\left(\left(\frac{a}{b}\right)^{\frac{q}{2}}, \lambda\right)\right\},
\end{aligned}
$$

where $h$ is defined as in (10). 
Proof Let $f(x)=\frac{x^{n+1}}{n+1}, x>0, n>0$ and $q \geq 1$. Then the function $\left|f^{\prime}(x)\right|^{q}=x^{n q}$ is quasigeometrically convex on $(0, \infty)$. Thus, by inequality (11), Proposition 3.1 is proved.

Proposition 3.2 For $b>a>0, n>0$ and $q>1$, we have

$$
\begin{aligned}
\left|(1-\lambda) G^{n+1}(a, b)+\lambda A\left(a^{n+1}, b^{n+1}\right)-(n+1) L(a, b) L_{n}^{n}(a, b)\right| \\
\leq \frac{n+1}{2}\left(\frac{\ln \frac{b}{a}\left(\lambda^{p+1}+(1-\lambda)^{p+1}\right)}{2(p+1)}\right)^{\frac{1}{p}}\left\{a G^{n}(a, b)\left(\frac{G^{q}(a, b)-a^{q}}{q}\right)^{\frac{1}{q}}\right. \\
\left.\quad+b^{n+1}\left(\frac{b^{q}-G^{q}(a, b)}{q}\right)^{\frac{1}{q}}\right\} .
\end{aligned}
$$

Proof Let $f(x)=\frac{x^{n+1}}{n+1}, x>0, n>0$ and $q>1$. Then the function $\left|f^{\prime}(x)\right|^{q}=x^{n q}$ is quasigeometrically convex on $(0, \infty)$. Thus, by inequality (13), Proposition 3.2 is proved.

\section{Competing interests}

The author declares that he has no competing interests.

\section{Received: 4 August 2013 Accepted: 9 September 2013 Published: 07 Nov 2013}

\section{References}

1. Ion, DA: Some estimates on the Hermite-Hadamard inequality through quasi-convex functions. An. Univ. Craiova, Ser. Mat. Inform. 34, 82-87 (2007)

2. Niculescu, CP: Convexity according to the geometric mean. Math. Inequal. Appl. 3(2), 155-167 (2000). doi:10.7153/mia-03-19

3. Niculescu, CP: Convexity according to means. Math. Inequal. Appl. 6(4), 571-579 (2003) doi:10.7153/mia-06-53

4. Zhang, T-Y, Ji, A-P, Qi, F: On integral inequalities of Hermite-Hadamard type for s-geometrically convex functions. Abstr. Appl. Anal. 2012, Article ID 560586 (2012). doi:10.1155/2012/560586

5. Kilbas, AA, Srivastava, HM, Trujillo, JJ: Theory and Applications of Fractional Differential Equations. Elsevier, Amsterdam (2006)

6. Alomari, M, Darus, M, Dragomir, SS, Cerone, P: Ostrowski type inequalities for functions whose derivatives are s-convex in the second sense. Appl. Math. Lett. 23, 1071-1076 (2010)

7. Avci, M, Kavurmaci, H, Ozdemir, ME: New inequalities of Hermite-Hadamard type via s-convex functions in the second sense with applications. Appl. Math. Comput. 217, 5171-5176 (2011)

8. Dahmani, Z: On Minkowski and Hermite-Hadamard integral inequalities via fractional via fractional integration. Ann. Funct. Anal. 1(1), 51-58 (2010)

9. Iscan, I: A new generalization of some integral inequalities for $(\alpha, m)$-convex functions. Math. Sci. 7, 22 (2013). doi:10.1186/2251-7456-7-22

10. Iscan, I: New estimates on generalization of some integral inequalities for $(\alpha, m)$-convex functions. Contemp. Anal. Appl. Math. 1(2), 253-264 (2013)

11. Iscan, I: New estimates on generalization of some integral inequalities for s-convex functions and their applications. Int. J. Pure Appl. Math. 86(4), 727-746 (2013)

12. Iscan, I: On generalization of some integral inequalities for quasi-convex functions and their applications. Int. J. Eng Appl. Sci. 3(1), 37-42 (2013)

13. Park, J: Generalization of some Simpson-like type inequalities via differentiable $s$-convex mappings in the second sense. Int. J. Math. Math. Sci. 2011, Article ID 493531 (2011). doi:10.1155/493531

14. Sarıkaya, MZ, Aktan, N: On the generalization of some integral inequalities and their applications. Math. Comput. Model. 54, 2175-2182 (2011)

15. Set, E: New inequalities of Ostrowski type for mapping whose derivatives are $s$-convex in the second sense via fractional integrals. Comput. Math. Appl. 63, 1147-1154 (2012)

16. Sarıkaya, MZ, Ogunmez, H: On new inequalities via Riemann-Liouville fractional integration. Abstr. Appl. Anal. 2012, Article ID 428983 (2012). doi:10.1155/2012/428983

17. Set, E, Ozdemir, ME, Sarıkaya, MZ: On new inequalities of Simpson's type for quasi-convex functions with applications Tamkang J. Math. 43(3), 357-364 (2012)

18. Sarıkaya, MZ, Set, E, Ozdemir, ME: On new inequalities of Simpson's type for s-convex functions. Comput. Math. Appl. 60, 2191-2199 (2010)

19. Sarıkaya, MZ, Set, E, Yaldız, H, Başak, N: Hermite-Hadamard's inequalities for fractional integrals and related fractional inequalities. Math. Comput. Model. 57(9-10), 2403-2407 (2013)

20. Zhu, C, Feckan, M, Wang, J: Fractional integral inequalities for differentiable convex mappings and applications to special means and a midpoint formula. J. Appl. Math. Stat. Inform. 8, 21-28 (2012)

21. Abramowitz, M, Stegun, IA (eds.): Handbook of Mathematical Functions with Formulas, Graphs and Mathematical Tables. Dover, New York (1965) 
10.1186/1029-242X-2013-491

Cite this article as: İscan: New general integral inequalities for quasi-geometrically convex functions via fractional integrals. Journal of Inequalities and Applications 2013, 2013:491

Submit your manuscript to a SpringerOpen ${ }^{\circ}$ journal and benefit from:

- Convenient online submission

- Rigorous peer review

- Immediate publication on acceptance

- Open access: articles freely available online

- High visibility within the field

- Retaining the copyright to your article 